\title{
Association of a Critical CSF Tryptophan Threshold Level with Depressive Relapse
}

\author{
Ronald M Salomon*,', John S Kennedy², Benjamin W Johnson³, Dennis E Schmidt', Joseph Kwentus', Harry E \\ Gwirtsman' and Michael H Ebert' \\ 'Department of Psychiatry, Vanderbilt University School of Medicine, Nashville, TN, USA; ${ }^{2}$ Department of Psychiatry, Indiana University School of \\ Medicine, Indianapolis, IN, USA; ${ }^{3}$ Department of Anesthesiology, Vanderbilt University School of Medicine, Nashville, TN, USA
}

\begin{abstract}
This work studies association between relapse during acute tryptophan depletion (ATD) and CSF level of tryptophan (TRP) in remitted depressives treated with sertraline or bupropion. Eight medication-responding depressives ingested an ATD amino acid mixture during 48-h continuous CSF sampling before and after treatment. Mood rating scores were compared with nadir levels of TRP in CSF. CSF TRP nadirs averaged $8.7 \%$ of am baselines in remitted patients. Mood relapsed whenever the CSF nadir was below $40 \mathrm{nmol} / \mathrm{I}$ TRP in remitted patients, and never when above (Fisher's exact test, $P=0.029$ ). Relapsing medication responders also showed very low preantidepressant ATD-induced nadirs. ATD-induced relapses were associated with low CSF TRP levels. Individual susceptibility to depletion may be independent of antidepressant treatment, mood state, or treatment status. Resistance to relapse may invoke an undefined, protective CNS mechanism against extremely low CSF levels of TRP during ATD.

Neuropsychopharmacology (2003) 28, 956-960, advance online publication, 19 March 2003; doi: I 0. I038/sj.npp. I 300098
\end{abstract}

Keywords: depression; 5-HIAA; serotonin; tryptophan; CSF; antidepressive agents

\section{INTRODUCTION}

Most remitted depressive patients experience a brief mood relapse during acute tryptophan depletion (ATD). The mood change is weakly, if at all, correlated with the observed $80-90 \%$ drop in plasma tryptophan (TRP). Instead, vulnerability to relapse has been associated with various symptom clusters (Delgado et al, 1990, 1991, 1994, 1999; Salomon et al, 1992, 1993; Benkelfat et al, 1995; Leyton et al, 1997; Lenzinger et al, 1999; Moreno et al, 2000a; Neumeister et al, 2002; Moore et al, 2000). ATD does not affect mood in healthy subjects (Abbott et al, 1992; Salomon et al, 1997). CSF TRP concentrations during ATD in healthy subjects are not associated with mood changes (Carpenter et al, 1998; Williams et al, 1999; Moreno et al, 2000b). An explanation for individual vulnerability to relapse is lacking.

Depressed patients were TRP depleted before and then after treatment with sertraline or bupropion. CSF TRP levels

Presented, in part, in meeting abstracts at APA, Toronto, May 1998, and American College of Neuropsychopharmacology, Puerto Rico, December 1998.

*Correspondence: Dr RM Salomon, Department of Psychiatry, Vanderbilt University School of Medicine, 150021 st Avenue South, VAV 2200, Nashville, TN 37212, USA, Fax: +I 6153439038 , E-mail: ron.salomon@vanderbilt.edu

Received 25 January 2002; revised 31 July 2002; accepted 18 October 2002

Online publication: 23 October 2002 at http://www.acnp.org/citations/ Npp I023024I5 were measured continuously, sampling every $10 \mathrm{~min}$ through the ATD nadir period. We hypothesized that sertraline-remitted patients would relapse if a critically low TRP level was reached, and that bupropion responders would be protected during ATD. However, our preliminary data suggest that CSF TRP nadir levels influence mood state more than the medication used. There may be a metabolic vulnerability, or absence of depletion-protective mechanism, in a subset of depressive individuals.

\section{METHODS}

Vanderbilt's Institutional Review Board and the General Clinical Research Center (GCRC) committee approved this protocol and associated advertisements (1996-1999). Patients consented after lengthy discussions of procedures, potential risks, and concerns. Depressed (ages 18-65, Hamilton rating scale for depression (HRSD) $\geqslant 18$ at screening, 2 months antidepressant free) medically healthy patients (by thorough laboratory, neurological, and physical exams) were eligible. Exclusions included suicidality, psychosis, tobacco use or nonaffective primary psychiatric diagnosis, or past severe headache, back problems, serious illness, or severe suicide attempt. Family and psychiatric histories were recorded.

Major depressive episode (DSM-IV, APA, 1994) was diagnosed clinically and by SCID-I/P and SCID-II (First et al, 1996a,b). The HRSD (Mazure et al, 1986) defined eligibility (17-item, $\geqslant 18$ ), remission (decrease $50 \%$ or $\leqslant 8$ ), and ATD relapse (10 point rise or $\geqslant 18)$. Adverse events 
were recorded twice daily. Balanced monoamine meals began 3 days before continuous CSF collection and excluded methylxanthines. Rooms were darkened from 23:00 to $06: 15$. ATD (using the amino acid mixture described by Delgado et al, 1990) began at 09:30 after $25.5 \mathrm{~h}$ of baseline CSF sampling. The study design did not include the placebo drink.

Pretreatment 48-h continuous CSF collections preceded 8 weeks of open pharmacotherapy (sertraline $50 \mathrm{mg}$ daily week one, then $100 \mathrm{mg}$; or bupropion $150 \mathrm{mg}$ daily week one, then $150 \mathrm{mg}$ BID). A second continuous CSF collection followed the fifth week of treatment. This monotherapy (except for one patient who continued her lithium throughout) was monitored for 3 weeks beyond the second CSF collection.

Antecubital venous plasma was frozen $\left(-60^{\circ} \mathrm{C}\right)$ for later assay of 'Total TRP'. Plasma ultrafiltrate (Amicon, Beverly, MA) was frozen for nonprotein-bound 'Free TRP' assays. Lumbar catheters were placed (Bruce and Oldfield, 1988; Kennedy et al, 1999) before 07:30, after $12 \mathrm{~h}$ supine, and collections began at 08:00. CSF was collected continuously, in 10-min aliquots, in polypropylene $\left(4^{\circ} \mathrm{C}\right)$, moved to dry ice within $30 \mathrm{~min}$, and then stored $\left(-60^{\circ} \mathrm{C}\right)$.

A previously reported reductive electrochemical HPLC method was modified to measure TRP in plasma and CSF and 5-HIAA in CSF (Schmidt et al, 1990). 5-HIAA and TRP assays were stable across the 3-year study period. Interassay (between day) coefficients of variance for both TRP and 5HIAA were $7.9 \%$ (144 replicate analyses of pooled CSF over 4 years on three different HPLC systems). 'Within assay' coefficients of variance, run on 288 replicates over 4 days, were $1.5 \%$ for TRP and $1.8 \%$ for 5-HIAA. Minimum sensitivity was 0.01-0.002 times the measured amounts.

Independent, blind analyses were conducted. Mood relapse and ATD nadirs (absolute lowest value) were analyzed for association ( $n=8$, all two-tailed tests, all $a$ priori specified $\alpha=0.05$ ). The 24 -h mean pre-ATD levels $(n=144)$, termed 'baseline', were compared with ATD nadir values to determine percent declines. The percent decline was also expressed relative to the 8:00 am pre-ATD level, for comparison with values in the literature (see abstract). Lithium treatment in one patient did not affect TRP levels.

Fisher's exact test assessed categorical comparisons. Differences between group means for continuous measurements were tested by the Student's $t$-test and checked by the Mann-Whitney $U$ test. Before-after comparisons were analyzed with the paired $t$-test and checked with the Wilcoxon signed-rank test. Spearman correlation was used to assess the relation between continuous variables. Statistical analyses were performed on using SPSS for Windows (Version 10.0, SPSS, Chicago).

\section{RESULTS}

A total of 16 consenting patients entered, 12 remained after all assessment procedures, and one was released before CSF collection because of a 'dry tap'. Of these, 11 (mean \pm SD, range: age $36.6 \pm 9.1$ years, $26-50 ; 82 \%$ females; HRSD $19.4 \pm 4.4,15-27)$ completed the study procedures. As responders to antidepressant treatment, eight (seven females) were the a priori intended focus of study. Treatment groups (sertraline $v s$ bupropion) were indis- tinguishable (NS) in baseline mood ratings, mean (24-h baseline) plasma free and total TRP levels, and CSF 5-HIAA levels.

Treatment compliance was assessed by interview only. No baseline measure predicted antidepressant response. Treatment assignment did not predict levels of CSF or plasma TRP (free or total), or CSF 5-HIAA (Fisher's exact test, NS). Symptoms remitted in five of seven sertraline-treated patients and three of four bupropion-treated patients. During ATD, depressive relapses were brief and without clinical consequence. There were no significant adverse events and no withdrawals for any reason. Of 26 physical symptom checklist (PSC) items, only memory, drowsiness, and trouble concentrating averaged in the mild to moderate range at any time point, and this was at baseline, improving over time. No group-averaged mean symptom score was ever significantly worse than at baseline (PSC, paired $t$-test, $\alpha 0.05)$. One patient, on one occasion several hours after ingesting the amino acid mixture, vomited without sequellae. Baseline headache ratings were mild to moderate (rated $1-4,2.2 \pm 0.9$ ), so that significant worsening would be expected only if severe headaches dominated. Severe headaches were unusual, any headache was infrequent, and all postspinal headaches resolved immediately (after blood patch in five of 26 catheterizations including four non-ATD studies).

\section{Treatment Effects - Findings from Baseline Sampling, before ATD}

Baseline CSF 5-HIAA levels (24-h means of 144 samples per study, before ATD) declined with treatment, as expected (cf Agren, 1980, $P=0.025, n=11$ ). This was usually observed in sertraline-treated patients (mean $67.5 \%$ of pretreatment, $n=7, P=0.02$ ) but only some bupropiontreated patients $(94.0 \%$ of pretreatment, $n=4$, NS). Withinsubjects CSF TRP baseline levels were highly correlated between studies $(r=0.85, n=11)$, as observed in both the sertraline and bupropion groups. Correlations were weak between mean CSF TRP and mean 5-HIAA, mean plasma total TRP, or mean plasma free TRP, both before and after treatment.

\section{Treatment Responders-Findings from Baseline Sampling, before ATD}

Among responders $(n=8)$, treatment was associated with declines in mean ( $n=144$ samples per study) CSF 5-HIAA and plasma total TRP (76.0\% (pre- to postantidepressant, $P=0.03)$ and $77.0 \%(P=0.05)$, respectively). Free plasma TRP and CSF TRP were not significantly changed with treatment $(n=8)$.

\section{ATD Findings}

Preantidepressant ATD was associated with irritability in the lithium-maintained patient (HRSD rise from screening to first ATD nadir, 3 points). She also showed the only preantidepressant CSF TRP nadir $(19.5 \mathrm{nM} / \mathrm{l})$ below $40 \mathrm{nM} / \mathrm{l}$. No clinically notable mood change was observed before antidepressant treatment. Pretreatment CSF TRP nadirs correlated most strongly with free plasma TRP $(r=0.81$, 
$\mathrm{df}=6, P=0.025$, two-tailed) but weakly with total plasma TRP $(r=0.37$, NS) and very weakly with CSF 5-HIAA $(r=0.16, \mathrm{NS})$ nadirs. Post-treatment ATD was the focus of the study in the medication responders $(n=8$, sertralinetreated $n=5$, bupropion-treated $n=3$ ). The ATD was associated with mood relapse after sertraline treatment $(60 \%$, HRSD change mean $=-9.7$ points $)$ and also in a bupropion-remitted (33\%, change $=-11$ points) patient. Most nonrelapsing patients showed small rises in HRSD (mean change $=+2.75$ ), suggesting a bimodal mood response to ATD. After treatment, the CSF TRP nadir correlated weakly and inversely with CSF 5-HIAA $(r=-0.24$, NS), plasma total TRP $(r=-0.03, \mathrm{NS})$ but tended towards significance with free plasma TRP nadirs $(r=-0.64, \mathrm{df}=6, P=0.1)$.

All TRP and 5-HIAA levels declined with ATD (all $P=0.0001)$. CSF TRP nadir ranges were from 1.7 to $39.9 \%$ (first ATD) or 1.6 to $8.7 \%$ (second ATD) of respective baselines $(n=8$; both $P<0.0001)$. CSF 5 -HIAA levels also declined from the respective baselines in both studies $(51.3 \% \pm 22.7$ of first ATD baseline; $52.7 \% \pm 10.9$ of second baseline, $n=8$, both $P<0.0001)$. Mood HRSD scores during the second (remitted baseline) ATD $(n=8)$ were correlated very weakly with 5 -HIAA $(r=0.18$, NS), plasma free TRP $(r=0.36$, NS), or plasma total TRP $(r=0.07$, NS), but strongly and inversely with CSF TRP $(r=-0.784, P=0.03)$. CSF TRP nadirs lasted for an average of $3.2 \mathrm{~h}$ (defined as within $5 \%$ of baseline from nadir, $n=21$ studies, $\mathrm{SD}=0.93 \mathrm{~h}$ ).

Mood relapsed in all remitted patients with a CSF TRP nadir below $40 \mathrm{nM}(n=4$, all female), and none of the patients $(n=4$, three female) above that level $(P=0.029$, Fisher's exact test, see Figure 1). Three of four relapsing patients also had the lowest levels of CSF TRP during the pretreatment ATD. Percent decline in CSF TRP from baseline (the lowest $=1.2 \%$ ) was not associated with changes in mood measures (Table 1).

TRP nadirs for relapsing and non-relapsing patients Median $+/$ - interquartile range, $n=4$ in each group

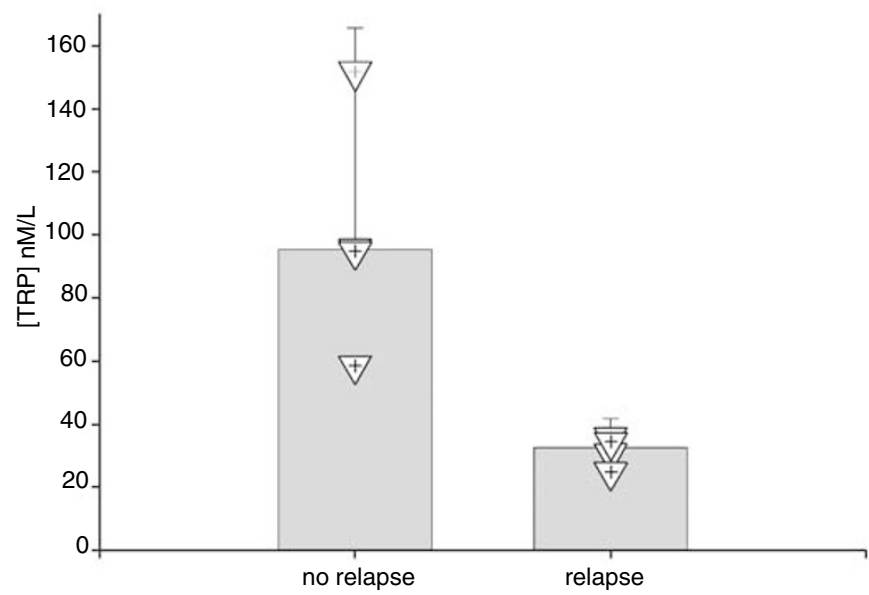

Figure I Median and quartile excursions for small $n$ groups. Individual points marked by open triangle symbols (note: close overlap of points (95.0 and 95.7) in nonrelapsed group may be difficult to distinguish as 2 points). (*Fisher's exact test two-tailed, $n=4$ in each group; $P=0.029$.)

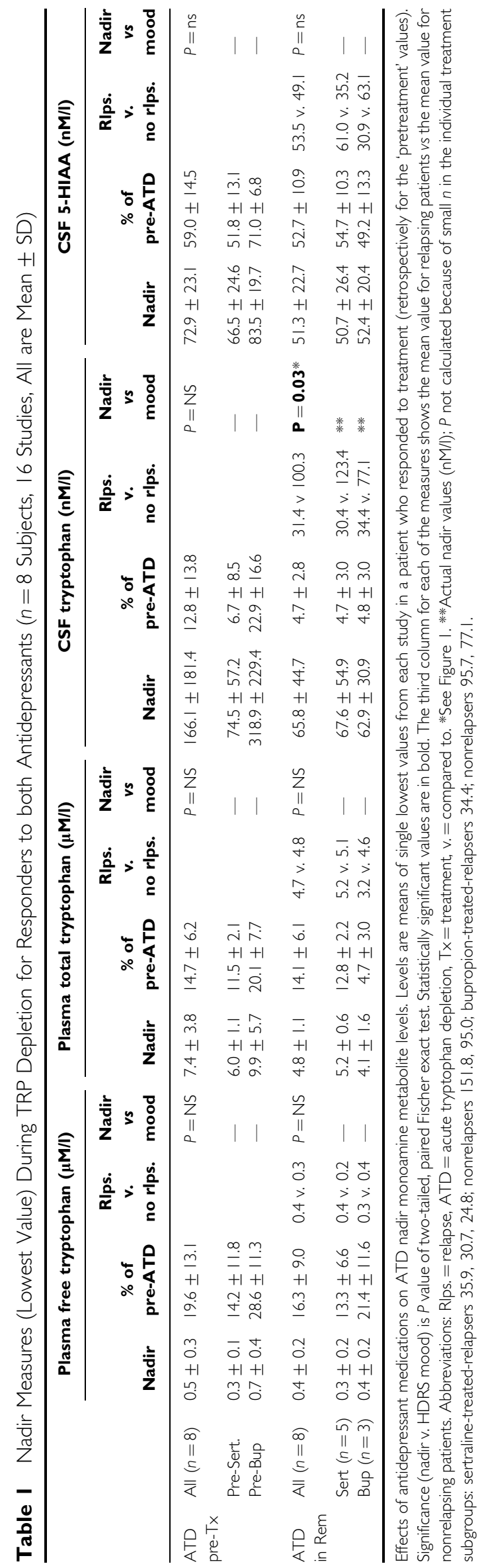




\section{DISCUSSION}

Only about half of the remitted depressives relapse briefly during ATD (Delgado et al, 1990). The present preliminary data associate relapse with a threshold TRP level in CSF. TRP levels in four patients' lumbar CSF dropped below $40 \mathrm{nM} / 1$ and all relapsed, while four others neither reached the critical level nor showed a mood relapse. Since this subset of depressives also showed the lowest TRP nadirs prior to antidepressant treatment, abnormal indoleamine metabolism may be a stable trait among those who relapse. However, even if an ATD-relapsing subset is identified, only remission-related inferences can be drawn since ATD prior to remission caused a worsening of symptoms only in one patient, who was maintained throughout the study on her chronic lithium regimen, and who also showed the only preantidepressant subthreshold TRP nadir.

Frequent (every $10 \mathrm{~min}$ ) serial measurements of CSF TRP and 5-HIAA levels assured a sample within $5 \mathrm{~min}$ of the absolute nadir, while this was not true for plasma (highest frequency every $30 \mathrm{~min}$ ) and might account for poor plasma TRP-mood correlations. However, the CSF nadirs remained extremely low for more than $1 \mathrm{~h}$, so the plasma sampling frequency should not have been an obstacle. Findings with the mechanistically distinct, catecholaminergic antidepressant bupropion (Ascher et al, 1995; Ferris et al, 1983; Dong and Blier, 2001) were not distinguished from those with the SSRI sertraline.

Unusually low CSF TRP levels may precipitate a change in mood, presumably through depletion of rapid-turnover pools of serotonin (5-HT). 5-HIAA declines were not correlated with mood or CSF TRP level, but were delayed (hours) compared to the rapid decline in CSF TRP. Lumbar CSF circulation may reflect central TRP levels rapidly, having the same plasma source, while rostral 5-HIAA mixes and flows slowly, delaying and dampening significance of the signal. Besides a direct effect on 5-HT, other explanations might include kynurenine metabolism (Moroni, 1999) or indirect neuronal network effects. Further study is needed to elucidate the mechanisms in this vulnerable patient subset.

\section{Limitations}

Continuous CSF sampling in our hands is well tolerated, but its demands, and hence the small $n$, limit generalizability. Gender may be an important source of variability of response to ATD, and was not equitably balanced in this study. Increased sensitivity to TRP depletion in depressed women has been reported, and decreased brain uptake of [11-C] $\alpha$-methyl-tryptophan (Nishizawa et al, 1997) was observed in healthy female subjects after TRP depletion. Our subject numbers are too small and predominantly female to draw any gender-based conclusions. However, females showed both the vulnerable and nonvulnerable response in an almost $1: 1$ ratio. The complexity and potential risks posed by this procedure limit the study size to a minimum that contributes a statistically valid sample. Conclusions regarding gender specificity, or past psychiatric or family history relations with relapse potential would require a larger sample.

\section{Summary}

Mixed responses to ATD among remitted patients may be because of central nervous system vulnerability to, or to lacking protection from a depleted plasma source of the 5HT precursor, TRP. This data offers a putative phenotype that may predispose to a depression subtype. The biochemical response to depletion of the 5-HT precursor appears to be an individual trait, not a mood or treatment state. This does not imply that 5-HT is not linked to mood in the nonrelapsing patients, but that they are able to compensate for dietary depletions of TRP. Further study, when new methods are available to follow TRP fate and metabolism rates, will be needed to confirm the present suggested threshold and better understand TRP depletion.

\section{ACKNOWLEDGEMENTS}

We gratefully acknowledge assistance and comments from Sidney Spector, $\mathrm{PhD}$, Fridolin Sulser, MD, and Pedro L Delgado, MD in early planning and execution of this work; support of the Vanderbilt General Clinical Research Center through a grant from NIH/HCRR (MO1RR00095) awarded to Vanderbilt University Medical Center, Nashville, TN; and for investigator-initiated funding from Pfizer, Inc. Statistical assistance from Daniel Byrne, MS at the Vanderbilt GCRC is greatly appreciated. Support from the Stanley Foundation and a NARSAD young investigator's award to RMS for parallel, closely related studies are also acknowledged and appreciated.

\section{REFERENCES}

Abbott FV, Etienne P, Franklin KB, Morgan MJ, Sewitch MJ, Young SN (1992). Acute tryptophan depletion blocks morphine analgesia in the cold-pressor test in humans. Psychopharmacology (Berl) 108: 60-66.

Agren H (1980). Symptom patterns in unipolar and bipolar depression correlating with monoamine metabolites in the cerebrospinal fluid: II. Suicide. Psychiatry Res 3: 225-236.

American Psychiatric Association (1994). Diagnostic and Statistical Manual of Mental Disorders, 4th edn. American Psychiatric Association: Washington, DC.

Ascher JA, Cole JO, Colin JN, Feighner JP, Ferris RM, Fibiger HC et al (1995). Bupropion: a review of its mechanism of antidepressant activity. J Clin Psychiatry 56: 395-401.

Benkelfat C, Seletti B, Palmour RM, Hillel J, Ellenbogen M, Young SN (1995). Tryptophan depletion in stable lithium-treated patients with bipolar disorder in remission. Arch Gen Psychiatry 52: $154-156$

Bruce JN, Oldfield EH (1988). Method for sequential sampling of cerebrospinal fluid in humans. Neurosurgery 23: 788-790.

Carpenter LL, Anderson GM, Pelton GH, Gudin JA, Kirwin PD, Price LH et al (1998). Tryptophan depletion during continuous CSF sampling in healthy human subjects. Neuropsychopharmacology 19: 26-35.

Delgado PL, Charney DS, Price LH, Aghajanian GK, Landis H, Heninger GR (1990). Serotonin function and the mechanism of antidepressant action. Reversal of antidepressant-induced remission by rapid depletion of plasma tryptophan. Arch Gen Psychiatry 47: 411-418.

Delgado PL, Miller HL, Salomon RM, Licinio J, Krystal JH, Moreno FA et al (1999). Tryptophan-depletion challenge in depressed patients treated with desipramine or fluoxetine: implications for 
the role of serotonin in the mechanism of antidepressant action. Biol Psychiatry 46: 212-220.

Delgado PL, Price LH, Miller HL, Salomon RM, Aghajanian GK, Heninger GR et al (1994). Serotonin and the neurobiology of depression. Arch Gen Psychiatry 51: 865-874.

Delgado PL, Price LH, Miller HL, Salomon RM, Licinio J, Krystal $\mathrm{JH}$ et al (1991). Rapid serotonin depletion as a provocative challenge test for patients with major depression: relevance to antidepressant action and the neurobiology of depression. Psychopharmacol Bull 27: 321-330.

Dong J, Blier P (2001). Modification of norepinephrine and serotonin, but not dopamine, neuron firing by sustained bupropion treatment. Psychopharmacology (Berl) 155: 52-57.

Ferris RM, Cooper BR, Maxwell RA (1983). Studies of bupropion's mechanism of antidepressant activity. J Clin Psychiatry 44: 74-78.

First MB, Spitzer RL, Gibbon M, Williams JBW (1996a). Structured Clinical Interview for DSM-IV Axis I Disorders (SCID-I/P) 2. Biometrics Research Department, New York State Psychiatric Institute: New York.

First MB, Spitzer RL, Gibbon M, Williams JBW, Benjamin L (1996b). Structured Clinical Interview for DSM-IV Axis II Disorders (SCID-II) 2. Biometrics Research Department, New York State Psychiatric Institute: New York.

Kennedy JS, Polinsky RJ, Johnson B, Loosen P, Enz A, Laplanche R et al (1999). Preferential cerebrospinal fluid acetylcholinesterase inhibition by rivastigmine in humans. J Clin Psychopharmacol 19: $513-521$.

Lenzinger E, Neumeister A, Praschak-Rieder N, Fuchs K, Gerhard E, Willeit $M$ et al (1999). Behavioral effects of tryptophan depletion in seasonal affective disorder associated with the serotonin transporter gene? Psychiatry Res 85: 241-246.

Leyton M, Young SN, Benkelfat C (1997). Relapse of depression after rapid depletion of tryptophan. Lancet 349: 1840-1841.

Mazure CM, Nelson JC, Price LH (1986). Reliability and validity of the symptoms of major depressive illness. Arch Gen Psychiatry 143: $35-39$.

Moore P, Landolt H-P, Seifritz E, Clark C, Bhatti T, Kelsoe J et al (2000). Clinical and physiological consequences of rapid tryptophan depletion. Neuropsychopharmacology 23: 601-622.

Moreno FA, Heninger GR, McGahuey CA, Delgado PL (2000a). Tryptophan depletion and risk of depression relapse: a prospective study of tryptophan depletion as a potential predictor of depressive episodes. Biol Psychiatry 48: 327-329.

Moreno FA, McGavin C, Malan P, Gelenberg AJ, Heninger GR, Mathe AA et al (2000b). Tryptophan depletion selectively reduces CSF 5-HT metabolites in healthy young men: results from single lumbar puncture sampling technique. Int $J$ Neuropsychopharmacology 3: 277-283.

Moroni F (1999). Tryptophan metabolism and brain function: focus on kynurenine and other indole metabolites. Eur $J$ Pharmacol 375: 87-100.

Neumeister A, Konstantinidis A, Stastny J, Schwarz M, Vitouch O, Willeit $M$ et al (2002). An association between serotonin transporter gene promotor polymorphism (5-HTTLPR) and behavioral responses to tryptophan depletion in healthy women with and without family history of depression. Arch Gen Psychiatry. 59: 613-620.

Nishizawa S, Benkelfat C, Young SN, Leyton M, Mzengeza S, de Montigny C et al (1997). Differences between males and females in rates of serotonin synthesis in human brain. Proc Natl Acad Sci USA 94: 5308-5313.

Salomon RM, Delgado PL, Miller HL, Charney DS (1992). Tryptophan depletion studies in psychiatric disorders. Psyche 1: 7-16.

Salomon RM, Miller HL, Delgado PL, Charney D (1993). The use of tryptophan depletion to evaluate central serotonin function in depression and other neuropsychiatric disorders. Int Clin Psychopharmacol 2(Suppl): 41-46.

Salomon RM, Miller HL, Krystal JH, Heninger GR, Charney DS (1997). Lack of behavioral effects of monoamine depletion in healthy subjects. Biol Psychiatry 41: 58-64.

Schmidt DE, Roznoski ML, Ebert MH (1990). Qualitative and quantitative HPLC analysis of monoamine neurotransmitters and metabolites in CSF and brain tissue using reductive electrochemical detection. Biomed Chromotogr 4: 215-221.

Williams WA, Shoaf SE, Hommer D, Rawlings R, Linnoila M (1999). Effects of acute tryptophan depletion on plasma and cerebrospinal fluid tryptophan and 5-hydroxyindoleacetic acid in normal volunteers. J Neurochem 72: 1641-1647. 